\title{
Two-Section Two-Zone Fluidized Bed Reactor fluid dynamics: experimental study, hydrodynamic modelling and CFD simulations
}

\author{
Ignacio Julián, Javier Herguido, Miguel Menéndez \\ CREG (Catálisis, Separaciones Moleculares e Ingeniería de Reactores) \\ Instituto de Investigación en Ingeniería de Aragón (I3A). \\ Universidad de Zaragoza, Mariano Esquillor s/n, 50018, Zaragoza, Spain. \\ Tel. +34-976762707, Fax+34-976762043, e-mail: ijulian@unizar.es
}

\begin{abstract}
A Two Zone Fluidized Bed Reactor (TZFBR) represents an effective solution to integrate reaction and catalyst regeneration in a single fluidized bed reactor. Reactor hydrodynamics of an improved TZFBR configuration, that incorporates a different cross-sectional area between zones to allow a better fluid dynamic control on each zone separately, is here investigated.
\end{abstract}

\section{Introduction}

The TZFBR (Figure 1.a) represents a multipurpose reactor to overcome process limitations in such heterogeneous catalytic reactions that involve fast catalyst deactivation due to carbon deposition over the catalyst active surface [1]. A separated gas inlet generates reactive and regenerative atmospheres inside the reactor, i.e. two zones, and the catalyst particles axial mixing provides chemical reaction and in-situ catalyst regeneration leading to a steadystate process operation [1]. The underlying bubbling regime of this unconventional fluidized bed determines the solids transfer between reactor zones and, therefore, the TZFBR performance [2]. The goal of this project is to gain insight into the hydrodynamic behaviour of an improved TZFBR configuration: the so called Two Section-TZFBR (TS-TZFBR, Figure 1.b), that allows low regenerative-to-reactive gas flows if required. For this purpose, three different techniques have been employed. Firstly, experimental fluidization measurements have been conducted in cold pseudo2D TS-TZFBR facilities to evaluate the effect of different reactor variables on the TS-TZFBR bubbling behaviour. Secondly, the average bubble size evolution along the vertical bed position, $d_{b}(z)$, has been modeled with a novel non-parametric correlation based on the Mori-Wen empirical equation [3]. Lastly, Computer Fluid Dynamic (CFD) simulations have been performed to predict the TS-TZFBR bubble characteristics. Simulations have been carried out using an Eulerian Eulerian model approach. The accuracy of the CFD model predictions has been evaluated with experimental bubbling statistic results. The ability of the model for predicting bubble size distributions, bubble velocity, bubbling density or aspect ratio makes this CFD code a powerful tool for the TZFBR scale up process.

\section{Experimental TS-TZFBR hydrodynamics}

Several particle type (inert glass beads, catalytic $\gamma$ $\mathrm{Al}_{2} \mathrm{O}_{3}$ and phosphorescent $\mathrm{SrAl}_{2} \mathrm{O}_{4}$ ) with different particle sizes, $d_{p}=[100-700] \mu m$, have been fluidized. Experimental high-definition video recordings (Figure 2.a) of various bubbling regimes on pseudo-2D TS-TZFBR have been processed with an in-house developed Matlab ${ }^{\circledR}$ 2010a code for image post-processing. A critical comparison among bubbling results from different experiments allowed the evaluation of the effect of several reactor variables on the TS-TZFBR hydrodynamic performance, e.g. axial immersed gas distributor position, relative gas velocity in each zone or tapered section angle between zones.

\section{TS-TZFBR bubble size evolution modeling}

None of the existing bubble size correlations is able to predict the bubble shrinkage at an intermediate bed height due to the coupled effect of the bed section enlargement and the addition of new small bubbles from the immersed gas distributor. The Julián-Herguido-Menéndez model (JHM) provides an accurate estimation of the bubble size evolution along the bed vertical position as a function of the tapered section angle $(\alpha)$, gas flow through both reactor inlets $\left(\mathrm{u}_{\mathrm{gas}}\right)$ and immersed distributor location $\left(\mathrm{z}_{\mathrm{dis}}\right)$. The JHM represents a modified version of the classical Mori-Wen (MW) bubble diameter correlation for narrow fluidized beds [3]. Coupling MW predictions for the straight column regions and a mass balance for the gas phase within the tapered section, the JHM model is able to predict $d_{b}(\mathrm{z})$ precisely in a wide range of operational 
conditions: $\mathrm{u}_{\mathrm{gas}}-\mathrm{u}_{\mathrm{mf}}=[5-25] \mathrm{cm} / \mathrm{s}, \alpha=\left[0^{\circ}-85^{\circ}\right]$, $\mathrm{u}_{\mathrm{mf}}=[1.2-20.5] \mathrm{cm} / \mathrm{s}$ and $\mathrm{z}_{\mathrm{dis}}=[0-2] \mathrm{cm}$ over the section change height.

\section{CFD simulation of TS-TZFBR dynamics}

The Eulerian-Eulerian model approach or TwoFluid Model (TFM) is here applied to simulate the macroscopic hydrodynamics inside a TS-TZFBR as a dense fluidized bed (Figure 2.b). The TFM depicts both particulate and gas phases as continuum interpenetrating media, where the volume of a phase cannot be occupied by others, under the assumption of the kinetic theory approach for the solid phase formulation. Mass, momentum and energy balances for each phase as well as an interphase momentum transfer correlation as a closure equation are simultaneously solved by the simulation tool (Ansys CFX 12.1) using the finite element approach. The solid-fluid interphase momentum exchange coefficient is here determined with the Gidaspow drag function. The computational domain mimics half of the symmetric experimental pseudo-2D facility and has been discretized into more than $10^{5}$ tetrahedra. Adaptative time steps around $10^{-4}$ seconds have been used to ease the problem convergence.

\section{Results}

Experimental hydrodynamic results illustrate how the bubbling regime is influenced by the tapered section angle and the immersed distributor location (Figure 2.c). Gas flows through the lower and upper distributors can be adjusted to avoid short-circuit in the solid recirculation due to 'slugging' effects in the narrower bed section. The average bubble size shrinkage within the tapered region, which is related to the section enlargement and the addition of small new bubbles, could be accurately predicted by the
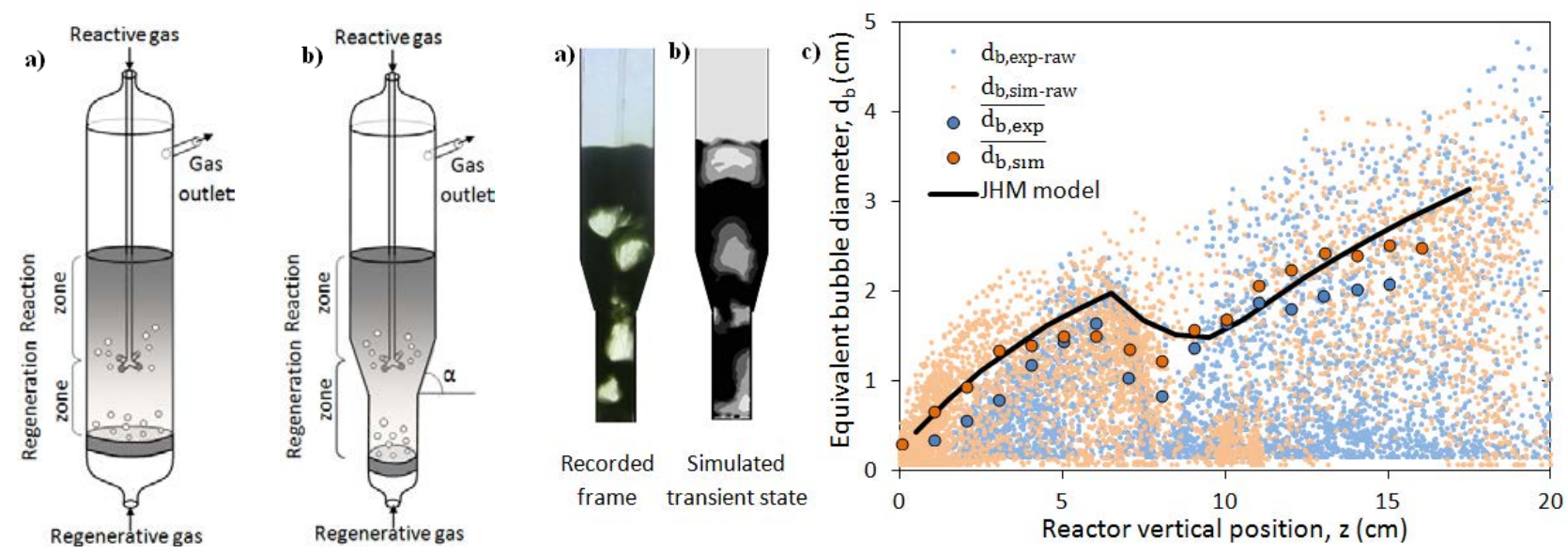

Figure 2. a) TS-TZFBR fluidization frame, b) TS-TZFBR transient CFD result, c) Axial bubble size evolution: experimental vs. simulated vs. JHM modeled developed JHM model spplying a mass balance to the gas phase in that region. Experimental bubbling results validated CFD model simulations in terms of bubble size evolution (Figure 2.c) for a wide range of fluidization gas flows rates. Further experimental bubble characteristics such bubble velocity, aspect ratio, bubble size distribution or bubbling frequency were reasonably well predicted by the computational model for the basis operational conditions: $\mathrm{u}_{\mathrm{gas}, \mathrm{l}} / \mathrm{u}_{\mathrm{mf}, \mathrm{l}}=\mathrm{u}_{\mathrm{gas}, \mathrm{u}} / \mathrm{u}_{\mathrm{mf}, \mathrm{u}}=2.5, \alpha=0^{\circ}$ and $\mathrm{z}_{\mathrm{dis}}=0$ centimeters over the section change location.

Most important findings of this research are: a) the development of an image processing algorithm for the detection and analysis of bubbles in a TSTZFBR b) the development of a non-parametric mathematical model to predict the bubble size evolution along the vertical bed position, taking into account the effect of the different fluid dynamic TSTZFBR system variables; c) the implementation of a CFD model which is able to simulate the lab-scale TS-TZFBR hydrodynamics accurately.

\section{REFERENCES}

[1] Herguido, J., Menéndez, M., and Santamaría, J. 2005. On the use of fluidized bed catalytic reactors where reduction and oxidation zones are present simultaneously, Catal. Today 100, 1-2 (Feb. 2005), 181-189. DOI=

http://dx.doi.org/10.1016/j.cattod.2004.11.004.

[2] Julián, I., Gallucci, F., van Sint Annaland, M., Herguido, J., Menéndez, M. 2012 Coupled PIV/DIA for fluid dynamic studies on a Two-Section Two-Zone Fluidized Bed Reactor, Chem. Eng. J. 207-208, (Oct. 2012), 122-132. DOI= http://dx.doi.org/10.1016/j.cej.2012.06.015.

[3] Mori, S., Wen, C.Y., 1975 Estimation of bubble diameter in gaseous fluidized beds, AIChE J. 21, 1 (Jan. 2011), 109 -115. DOI= http://dx.doi.org/10.1002/aic.690210114.

\section{Conclusions}

